\title{
KIRCHHOFF ELASTIC RODS IN A RIEMANNIAN MANIFOLD
}

\author{
SATOSHI KAWAKUBO
}

(Received May 10, 2000, revised January 22, 2001)

\begin{abstract}
Imagine a thin elastic rod like a piano wire. We consider the situation that the elastic rod is bent and twisted and both ends are welded together to form a smooth loop. Then, does there exist a stable equilibrium? In this paper, we generalize the energy of uniform symmetric Kirchhoff elastic rods in the 3-dimensional Euclidean space to consider such a variational problem in a Riemannian manifold. We give the existence and regularity of minimizers of the energy in a compact or homogeneous Riemannian manifold.
\end{abstract}

1. Introduction. Let $\mathcal{M}$ be an $n$-dimensional $C^{\infty}$ Riemannian manifold. Let $l$ be a positive constant, which represents the length of a piece of the elastic rod. We consider a unit-speed closed curve $\gamma=\gamma(t): S^{1}=\boldsymbol{R} / l \boldsymbol{Z} \rightarrow \mathcal{M}$. We assume that $\gamma$ is of class $H^{2}$, that is, the components of $\gamma$ with respect to any $C^{\infty}$ local coordinate system are of class $H_{\text {loc }}^{2}$ in $t$. To describe how the elastic rod is twisted, we consider an orthonormal frame field $M=\left(M_{1}, M_{2}, \ldots, M_{n-1}\right)$ in the normal bundle along $\left.\gamma\right|_{[0, l]}$. (In general, $M(0) \neq M(l)$.) Here, we assume that $M$ is of class $H^{1}$, that is, the components of $M_{i}(1 \leqslant i \leqslant n-1)$ with respect to any $C^{\infty}$ local coordinate system are of class $H_{\text {loc }}^{1}$ in $t$. We consider the pair $\{\gamma, M\}$ of $\gamma$ and $M$.

Let $v$ be a positive constant, which is determined by the material of the wire. We define the energy $\mathfrak{T}$ as follows:

$$
\mathfrak{T}(\{\gamma, M\})=\int_{0}^{l}\left|\nabla_{t} \dot{\gamma}\right|^{2} d t+v \sum_{i=1}^{n-1} \int_{0}^{l}\left|\nabla_{t}^{\perp} M_{i}\right|^{2} d t .
$$

This energy is a generalization of the energy of the uniform and symmetric case of Kirchhoff elastic rods in the 3-dimensional Euclidean space, which is possibly the simplest energy with the effect of bending and twisting (cf. [12], [5]). Here, the first term of the right hand side is called the bending energy of $\gamma$, which is the simplest energy with only the effect of bending.

We now formulate the space of configurations of the loop wire. We introduce an element $\varphi$ of the $(n-1)$-dimensional special orthogonal group $S O(n-1)$ to represent how the sides of the ends of the elastic rod are welded. Denote by $\mathcal{U C}(l, \varphi)$ the totality of the pair $\{\gamma, M\}$ as above satisfying the following boundary condition:

$$
M(l)=M(0) \varphi .
$$

Let $\mathcal{U C}_{0}(l, \varphi)$ be a connected component of $\mathcal{U C}(l, \varphi)$. This space represents the totality of configurations of the loop wire. We consider the following variational problem.

2000 Mathematics Subject Classification. Primary 58E10; Secondary 74K10. 
Problem. Does there exist an element of $\mathcal{U C}_{0}(l, \varphi)$ attaining the infimum of the energy $\mathfrak{T}$ ? Moreover, is the element smooth?

In the case that the ambient space is the Euclidean space, the existence and regularity theorem of minimizers was proved by Antman ([1]) for a much broader class of energies and boundary conditions. Also, in the case that the ambient space is a general compact Riemannian manifold and the energy is the bending energy, it was proved by Langer and Singer ([11]) and by Koiso ([9]). Langer and Singer ([11]) proved that the bending energy on the space of curves in a compact Riemannian manifold satisfies the Palais-Smale condition. This implies the existence of general critical curves including minimizers. Also, Koiso ([9]) proved the existence and regularity of minimizers of the bending energy on the space of curves restricted in a Riemannian submanifold by using the direct method of the calculus of variations.

In this paper, we affirmatively answer to the above variational problem in the case that the ambient space $\mathcal{M}$ is a compact or homogeneous Riemannian manifold and the energy is the $\mathfrak{T}$ defined above.

THEOREM 1.1. Suppose that $\mathcal{M}$ is a compact Riemannian manifold or a homogeneous Riemannian manifold. There exists $\{\gamma, M\} \in \mathcal{U C}_{0}(l, \varphi)$ attaining the infimum of the energy T. Moreover, $\{\gamma, M\}$ is of class $C^{\infty}$.

REMARK. Even for $\varphi \in O(n-1)$, the above theorem holds in the following sense. Namely, if $\mathcal{U C}(l, \varphi) \neq \emptyset$, then there exists a smooth minimizer of $\mathfrak{T}$ in any connected component $\mathcal{U} \mathcal{C}_{0}(l, \varphi)$ of $\mathcal{U C}(l, \varphi)$.

The organization of the paper is as follows: In Section 2, we introduce the notation precisely, and prove the existence of minimizers. In Section 3, we prove the regularity of minimizers.

The author would like to express his gratitude to Professor Norihito Koiso for his suggestion and constant encouragement. The author also wishes to thank Professor Minyo Katagiri for his advice and warm encouragement, and the referee for valuable comments.

2. Existence of minimizers. Let $\mathcal{M}$ be an $n$-dimensional $C^{\infty}$ Riemannian manifold, and $l$ a positive constant. For later convenience in Section 3, we define some configuration spaces consisting of those which are not necessarily parameterized by arc length. Let $\gamma=$ $\gamma(t): S^{1}=\boldsymbol{R} / l \boldsymbol{Z} \rightarrow \mathcal{M}$ be a regular closed curve. We assume that $\gamma$ is of class $H^{2}$, that is, the components of $\gamma$ with respect to any $C^{\infty}$ local coordinate system are of class $H_{\text {loc }}^{2}$ in $t$. Note that $\gamma$ is of class $C^{1}$ by the Sobolev embedding theorem. Let $\dot{\gamma}$ be the tangent vector to $\gamma$, and $v(t)=|\dot{\gamma}(t)|=\langle\dot{\gamma}(t), \dot{\gamma}(t)\rangle^{1 / 2}$ the speed, and $T(t)=(1 / v(t)) \dot{\gamma}(t)$ the unit tangent vector. We denote by $T \mathcal{M}$ the tangent bundle of $\mathcal{M}$, and by $\nabla$ the Levi-Civita connection. We use the symbols $\nabla_{t}=\nabla_{\partial / \partial t}=\nabla_{\dot{\gamma}}$. When we think of $\gamma$ as a curve having two end points $\gamma(0)$ and $\gamma(l)$, we denote it by $\left.\gamma\right|_{[0, l]}:[0, l] \rightarrow \mathcal{M}$. Denote by $T^{\perp} \mathcal{M}$ the normal bundle along $\left.\gamma\right|_{[0, l]}$, and by $\nabla^{\perp}$ the normal connection. Let $M=\left(M_{1}, M_{2}, \ldots, M_{n-1}\right)$ be an orthonormal frame field in $T^{\perp} \mathcal{M}$. (In general, $M(0) \neq M(l)$.) Here, we assume that $M$ is of class $H^{1}$, that is, for any $C^{\infty}$ local coordinate system $\left(x^{1}, x^{2}, \ldots, x^{n}\right)$, the components of 
$M_{i}(1 \leqslant i \leqslant n-1)$ with respect to the basis $\partial / \partial x^{1}, \partial / \partial x^{2}, \ldots, \partial / \partial x^{n}$ are of class $H_{\mathrm{loc}}^{1}$ in $t$. We consider the pair $\{\gamma, M\}$ of $\gamma$ and $M$.

Now, let $v$ be a positive constant. We define the energy $\mathfrak{T}$ as follows:

$$
\mathfrak{T}(\{\gamma, M\})=\int_{0}^{l}\left|\nabla_{T} T\right|^{2} v d t+v \sum_{i=1}^{n-1} \int_{0}^{l}\left|\nabla_{T}^{\perp} M_{i}\right|^{2} v d t .
$$

We note that $\mathfrak{T}$ is invariant under reparameterization of $t$.

Let $\varphi \in S O(n-1)$. Denote by $\mathcal{C}(\varphi)$ the totality of $\{\gamma, M\}$ as above satisfying the following boundary condition:

$$
M(l)=M(0) \varphi .
$$

Also, we denote by $\mathcal{C}(l, \varphi)$ the totality of elements $\{\gamma, M\}$ of $\mathcal{C}(\varphi)$ such that the length $\int_{0}^{l} v(t) d t$ of $\gamma$ is equal to $l$, and by $\mathcal{U C}(l, \varphi)$ the totality of elements $\{\gamma, M\}$ of $\mathcal{C}(l, \varphi)$ such that $\gamma$ is unit-speed.

Here, we introduce the following topology on $\mathcal{U C}(l, \varphi)$ to take its connected components. For a technical reason, we consider a slightly weaker topology than the natural topology on $\mathcal{U C}(l, \varphi)$. Note that the following inclusion relation holds.

$$
\begin{aligned}
\mathcal{U C}(l, \varphi) \subset \mathcal{C}(l, \varphi) \subset \mathcal{C}(\varphi) & \subset H^{2}\left(S^{1}, \mathcal{M}\right) \times\left(H^{1}([0, l], T \mathcal{M})\right)^{n-1} \\
& \subset C^{1}\left(S^{1}, \mathcal{M}\right) \times\left(C^{0}([0, l], T \mathcal{M})\right)^{n-1},
\end{aligned}
$$

where $\left(H^{1}([0, l], T \mathcal{M})\right)^{n-1}$, etc. are the $n-1$ times direct products of $H^{1}([0, l], T \mathcal{M})$, etc. We introduce on the right hand side the product topology of the $C^{1}$ topology of $C^{1}\left(S^{1}, \mathcal{M}\right)$ and the $C^{0}$ topology of $C^{0}([0, l], T \mathcal{M})$. We call it the $C^{1,0}$ topology. Let $\mathcal{U} \mathcal{C}_{0}(l, \varphi)$ be a connected component of $\mathcal{U C}(l, \varphi)$ with respect to the $C^{1,0}$ topology. (See the last paragraph of the proof of Lemma 2.1.)

Now, we prove Theorem 1.1. From now on, we assume that $\mathcal{M}$ is a compact or homogeneous Riemannian manifold. In the rest of this section, we show the existence of minimizers. Let $\left\{\gamma^{p}, M^{p}\right\}=\left\{\gamma^{p},\left(M_{1}^{p}, \ldots, M_{n-1}^{p}\right)\right\}(p=1,2, \ldots)$ be a minimizing sequence of $\mathfrak{T}$ in $\mathcal{U C}_{0}(l, \varphi)$, and $T_{0}$ the infimum of $\mathfrak{T}$. Thus, $\lim _{p \rightarrow \infty} \mathfrak{T}\left(\left\{\gamma^{p}, M^{p}\right\}\right)=T_{0}$.

LEMma 2.1. There exists a subsequence $\left\{\left\{\gamma^{p_{j}}, M^{p_{j}}\right\}\right\}_{j=1}^{\infty}$ of $\left\{\left\{\gamma^{p}, M^{p}\right\}\right\}_{p=1}^{\infty}$ and $\left\{\gamma^{\infty}, M^{\infty}\right\} \in \mathcal{U C} \mathcal{C}_{0}(l, \varphi)$ such that $\left\{\gamma^{p_{j}}\right\}$ converges to $\gamma^{\infty}$ in the weak topology of $H^{2}\left(S^{1}, \mathcal{M}\right)$ and $\left\{M^{p_{j}}\right\}$ converges to $M^{\infty}$ in the weak topology of $\left(H^{1}([0, l], T \mathcal{M})\right)^{n-1}$ as $j \rightarrow \infty$.

Proof. First, we consider the case that $\mathcal{M}$ is a homogeneous Riemannian manifold. Without loss of generality, we may assume that $\mathcal{M}$ is connected. Since $\mathcal{M}$ is connected, the connected component $G_{0}$ of the isometry group of $\mathcal{M}$ with the unit element also acts transitively on $\mathcal{M}$ as isometries. For $\sigma \in G_{0}$, we denote by $S_{\sigma}$ the corresponding isometry on $\mathcal{M}$, and by $S_{\sigma *}$ its differential map. If $\{\gamma, M\} \in \mathcal{U} \mathcal{C}_{0}(l, \varphi)$, then $\left\{S_{\sigma} \circ \gamma, S_{\sigma *} M\right\} \in \mathcal{U} \mathcal{C}_{0}(l, \varphi)$. Also, $\mathfrak{T}\left(\left\{S_{\sigma} \circ \gamma, S_{\sigma *} M\right\}\right)$ is equal to $\mathfrak{T}(\{\gamma, M\})$. Therefore, without loss of generality, we may assume that the points $\gamma^{p}(0)$ are independent of $p$. Thus, the images $\gamma^{p}\left(S^{1}\right)$ are contained in a compact subset of $\mathcal{M}$ independent of $p$. Next, in the case that $\mathcal{M}$ is a compact Riemannian 
manifold, the images $\gamma^{p}\left(S^{1}\right)$ are also contained in a compact set $\mathcal{M}$. (The compactness or homogeneity of $\mathcal{M}$ is not necessary, hereafter.)

By the condition $\left|\dot{\gamma}^{p}\right| \equiv 1$, the sequence $\left\{\gamma^{p}\right\}$ is equicontinuous. Thus, the Ascoli theorem ([7]) yields that there exists a subsequence $\left\{\gamma^{p_{j}}\right\}_{j=1}^{\infty}$ of $\left\{\gamma^{p}\right\}_{p=1}^{\infty}$ which converges to a closed curve $\gamma^{\infty} \in C^{0}\left(S^{1}, \mathcal{M}\right)$ in the $C^{0}$ topology. We rewrite the subsequence $\left\{\left\{\gamma^{p_{j}}, M^{p_{j}}\right\}\right\}_{j=1}^{\infty}$ as $\left\{\left\{\gamma^{p}, M^{p}\right\}\right\}_{p=1}^{\infty}$.

Let $I \subset S^{1}$ be an open interval, and $\left(U,\left(x^{1}, x^{2}, \ldots, x^{n}\right)\right)$ a local coordinate neighborhood of $\mathcal{M}$ satisfying $\gamma^{\infty}(\bar{I}) \subset U$, where $\bar{I}$ is the closure of $I$. For sufficiently large $p$, $\gamma^{p}(\bar{I}) \subset U$. Denote by $x \circ \gamma^{p}=\left(x^{1} \circ \gamma^{p}, x^{2} \circ \gamma^{p}, \ldots, x^{n} \circ \gamma^{p}\right)$ the coordinate expression of $\gamma^{p}$, and by $\left(M_{i}^{p}\right)^{1},\left(M_{i}^{p}\right)^{2}, \ldots,\left(M_{i}^{p}\right)^{n}$ the coordinate expression of $M_{i}^{p}$ with respect to the basis $\partial / \partial x^{1}, \partial / \partial x^{2}, \ldots, \partial / \partial x^{n}$. We show that

$$
\left\|x \circ \gamma^{p}\right\|_{H^{2}\left(I, \boldsymbol{R}^{n}\right)}^{2}=\int_{I}\left|x \circ \gamma^{p}\right|_{E}^{2}+\left|\left(x \circ \gamma^{p}\right)^{\prime}\right|_{E}^{2}+\left|\left(x \circ \gamma^{p}\right)^{\prime \prime}\right|_{E}^{2} d t
$$

is bounded with respect to $p$, where $|*|_{E}$ denotes the Euclidean norm of a vector in $\boldsymbol{R}^{n}$ and ' denotes differentiation with respect to $t$. First, $\left|x \circ \gamma^{p}\right|_{E}^{2}$ is bounded by a positive constant independent of $p$ and $t$, because $\left\{x \circ \gamma^{p}\right\}$ converges to $x \circ \gamma^{\infty}$ in the $C^{0}$ topology. Next, we consider $\left|\left(x \circ \gamma^{p}\right)^{\prime}\right|_{E}^{2}$. Since $\bigcup_{p=1}^{\infty} \gamma^{p}(\bar{I})(\subset \mathcal{M})$ is compact, there exists a positive constant $C_{1}$ independent of $p$ and $t$ such that

$$
\left|\left(x \circ \gamma^{p}\right)^{\prime}\right|_{E}^{2} \leqslant C_{1}\left|\dot{\gamma}^{p}\right|^{2}=C_{1} .
$$

Next, we consider

$$
\int_{I}\left|\left(x \circ \gamma^{p}\right)^{\prime \prime}\right|_{E}^{2} d t
$$

We denote by $\Gamma_{k l}^{i}$ the Christoffel symbols, and by $\left(\nabla_{t} \dot{\gamma}^{p}\right)^{1}, \ldots,\left(\nabla_{t} \dot{\gamma}^{p}\right)^{n-1}$ the components of $\nabla_{t} \dot{\gamma}^{p}$ with respect to the basis $\partial / \partial x^{1}, \ldots, \partial / \partial x^{n}$. Since

$$
\left(\nabla_{t} \dot{\gamma}^{p}\right)^{i}=\left(x^{i} \circ \gamma^{p}\right)^{\prime \prime}+\sum_{k, l=1}^{n} \Gamma_{k l}^{i}\left(x^{k} \circ \gamma^{p}\right)^{\prime}\left(x^{l} \circ \gamma^{p}\right)^{\prime},
$$

we have

$$
\begin{aligned}
\left|\left(x \circ \gamma^{p}\right)^{\prime \prime}\right|_{E}^{2} \leqslant & 2\left|\left(\left(\nabla_{t} \dot{\gamma}^{p}\right)^{1}, \ldots,\left(\nabla_{t} \dot{\gamma}^{p}\right)^{n}\right)\right|_{E}^{2} \\
& +2 \sum_{i=1}^{n}\left(\sum_{k, l=1}^{n} \Gamma_{k l}^{i}\left(x^{k} \circ \gamma^{p}\right)^{\prime}\left(x^{l} \circ \gamma^{p}\right)^{\prime}\right)^{2} \\
\leqslant & 2 C_{2}\left|\nabla_{t} \dot{\gamma}^{p}\right|^{2}+2 C_{3}\left|\left(x \circ \gamma^{p}\right)^{\prime}\right|_{E}^{4} \\
\leqslant & 2 C_{2}\left|\nabla_{t} \dot{\gamma}^{p}\right|^{2}+2 C_{3} C_{1}^{2},
\end{aligned}
$$

for almost every $t \in I$, where $C_{2}$ and $C_{3}$ are constants independent of $p$ and $t$. Therefore,

$$
\int_{I}\left|\left(x \circ \gamma^{p}\right)^{\prime \prime}\right|_{E}^{2} d t \leqslant 2 C_{2} \mathfrak{T}\left(\left\{\gamma^{p}, M^{p}\right\}\right)+2 C_{3} C_{1}^{2} l .
$$

Since $\mathfrak{T}\left(\left\{\gamma^{p}, M^{p}\right\}\right)$ is bounded by a constant independent of $p$, so is the left hand side of the above expression. 
Let $I_{1}, \ldots, I_{r} \subset S^{1}$ be a family of open intervals and $\left(U_{m},\left(x_{m}\right)\right)(m=1, \ldots, r)$ be a family of coordinate neighborhoods of $\mathcal{M}$ such that $S^{1}=I_{1} \cup \cdots \cup I_{r}$ and $\gamma^{\infty}\left(\overline{I_{m}}\right) \subset U_{m}$ for each $m$. For each $m,\left\|x_{m} \circ \gamma^{p}\right\|_{H^{2}\left(I_{m}, \boldsymbol{R}^{n}\right)}^{2}$ is bounded with respect to $p$. A bounded sequence in a Hilbert space has a weakly convergent subsequence ([2]). Thus, there exists a subsequence $\left\{\left\{\gamma^{p_{j}}, M^{p_{j}}\right\}\right\}_{j=1}^{\infty}$ such that $\left\{x_{m} \circ \gamma^{p_{j}}\right\}_{j=1}^{\infty}$ converges to an element $\xi_{m}$ of $H^{2}\left(I_{m}, \boldsymbol{R}^{n}\right)$ in the weak topology of $H^{2}\left(I_{m}, \boldsymbol{R}^{n}\right)$. For each $m$, we successively extract such a weakly convergent subsequence. We rewrite the resulting subsequence as $\left\{\left\{\gamma^{p}, M^{p}\right\}\right\}_{p=1}^{\infty}$. We can check that if $I_{k} \cap I_{m} \neq \emptyset$, then $\xi_{k}=\left(x_{k} \circ x_{m}^{-1}\right) \circ \xi_{m}$. Thus, there exists an element $\xi$ of $H^{2}\left(S^{1}, \mathcal{M}\right)$ such that $\xi_{m}=x_{m} \circ \xi$. Consequently, $\left\{\gamma^{p}\right\}$ converges to $\xi$ in the weak topology of $H^{2}\left(S^{1}, \mathcal{M}\right)$.

Since $H^{2}\left(I, \boldsymbol{R}^{n}\right)$ is compactly embedded in $C^{1}\left(I, \boldsymbol{R}^{n}\right)$, a weakly convergent sequence in $H^{2}\left(I, \boldsymbol{R}^{n}\right)$ is a strongly convergent sequence in $C^{1}\left(I, \boldsymbol{R}^{n}\right)$. Therefore, $\left\{\gamma^{p}\right\}$ converges to $\xi$ in the $C^{1}$ (strong) topology, and so $\xi=\gamma^{\infty}$. Also, we have $\left|\dot{\gamma}^{\infty}\right| \equiv 1$, because $\left\{\gamma^{p}\right\}$ converges to $\gamma^{\infty}$ in the $C^{1}$ topology.

Next, we consider $M_{i}^{p}$. We denote by $I$ an open interval in $[0, l]$. We write the pair of components $\left(\left(M_{i}^{p}\right)^{1},\left(M_{i}^{p}\right)^{2}, \ldots,\left(M_{i}^{p}\right)^{n}\right)$ as the same notation $M_{i}^{p}$, unless confusion could occur. We first show that $\left\|M_{i}^{p}\right\|_{H^{1}\left(I, \boldsymbol{R}^{n}\right)}$ is bounded with respect to $p$. Since $\left|M_{i}^{p}\right|^{2} \equiv 1$, it is sufficient to show that

$$
\int_{I}\left|\frac{d M_{i}^{p}}{d t}\right|_{E}^{2} d t
$$

is bounded with respect to $p$. In the same way as (2.1), we have

$$
\left|\frac{d M_{i}^{p}}{d t}\right|_{E}^{2} \leqslant C_{4}\left|\nabla_{t} M_{i}^{p}\right|^{2}+C_{5}
$$

for almost every $t \in I$, where $C_{4}$ and $C_{5}$ are constants independent of $p$ and $t$. By the Leibniz rule, $\left|\nabla_{t}^{\perp} M_{i}^{p}\right|^{2}=\left|\nabla_{t} M_{i}^{p}\right|^{2}-\left|\left\langle M_{i}^{p}, \nabla_{t} \dot{\gamma}^{p}\right\rangle\right|^{2}$, and so we have

$$
\sum_{i=1}^{n-1} \int_{I}\left|\nabla_{t} M_{i}^{p}\right|^{2} d t=\sum_{i=1}^{n-1} \int_{I}\left|\nabla_{t}^{\perp} M_{i}^{p}\right|^{2} d t+\int_{I}\left|\nabla_{t} \dot{\gamma}^{p}\right|^{2} d t \leqslant\left(1+\frac{1}{v}\right) \mathfrak{T}\left(\left\{\gamma^{p}, M^{p}\right\}\right) .
$$

Therefore, $\int_{I}\left|\nabla_{t} M_{i}^{p}\right|^{2} d t$ is bounded with respect to $p$, and so is (2.2).

In the same way as $\left\{\gamma^{p}\right\}$, we obtain the following. There exists a subsequence $\left\{\left\{\gamma^{p_{j}}, M^{p_{j}}\right\}\right\}_{j=1}^{\infty}$ such that $\left\{M_{i}^{p_{j}}\right\}_{j=1}^{\infty}\left(\subset H^{1}([0, l], T \mathcal{M})\right)$ converges to an element $M_{i}^{\infty}$ of $H^{1}([0, l], T \mathcal{M})$ in the weak $H^{1}$ topology of $H^{1}([0, l], T \mathcal{M})$. Thus, $\left\{M_{i}^{p_{j}}\right\}$ also converges to $M_{i}^{\infty}$ in the $C^{0}$ topology. We set $M^{\infty}=\left(M_{1}^{\infty}, \ldots, M_{n-1}^{\infty}\right)$. Since $M_{i}^{p_{j}}(t)$ converges to $M_{i}^{\infty}(t)$ and $\dot{\gamma}^{p_{j}}(t)$ converges to $\dot{\gamma}^{\infty}(t)$ for every fixed $t, M^{\infty}$ is an orthonormal frame field of the normal bundle along $\left.\gamma^{\infty}\right|_{[0, l]}$, and $M^{\infty}(l)=M^{\infty}(0) \varphi$. Therefore, $\left\{\gamma^{\infty}, M^{\infty}\right\} \in \mathcal{U C}(l, \varphi)$.

Here, recall that $\mathcal{U} \mathcal{C}_{0}(l, \varphi)$ is a connected component with respect to the $C^{1,0}$ topology. Since $\left\{\left\{\gamma^{p_{j}}, M^{p_{j}}\right\}\right\}_{j=1}^{\infty}$ converges to $\left\{\gamma^{\infty}, M^{\infty}\right\}$ in the $C^{1,0}$ topology, we have $\left\{\gamma^{\infty}, M^{\infty}\right\} \in$ $\mathcal{U C}_{0}(l, \varphi)$.

Now, we show $\mathfrak{T}\left(\left\{\gamma^{\infty}, M^{\infty}\right\}\right)=T_{0}$. We rewrite the subsequence $\left\{\left\{\gamma^{p_{j}}, M^{p_{j}}\right\}\right\}_{j=1}^{\infty}$ in Lemma 2.1 as $\left\{\left\{\gamma^{p}, M^{p}\right\}\right\}_{p=1}^{\infty}$. Let $0=t_{0}<t_{1}<\cdots<t_{r-1}<t_{r}=l$ be a subdivision 
of the interval $[0, l]$ and $\left(U_{m},\left(x_{m}\right)\right)(m=1, \ldots, r)$ be a family of coordinate neighborhoods of $\mathcal{M}$ such that $\gamma^{\infty}\left(\left[t_{m-1}, t_{m}\right]\right) \subset U_{m}$ for each $m$. We set $J_{m}=\left[t_{m-1}, t_{m}\right]$. We denote by $\left.\mathfrak{T}\right|_{J_{m}}(\{\gamma, M\})$ the integral of the corresponding energy density on $J_{m}$ instead of $[0, l]$ in the definition of $\mathfrak{T}$. Since the sequence $\left\{\left.\mathfrak{T}\right|_{J_{m}}\left(\left\{\gamma^{p}, M^{p}\right\}\right)\right\}_{p=1}^{\infty}$ is a bounded sequence of real numbers, there exists a convergent subsequence. Thus, without loss of generality, we may assume that the sequence $\left\{\left.\mathfrak{T}\right|_{J_{m}}\left(\left\{\gamma^{p}, M^{p}\right\}\right)\right\}_{p=1}^{\infty}$ converges to a real number for each $m$. Set

$$
T_{J_{m}}=\left.\lim _{p \rightarrow \infty} \mathfrak{T}\right|_{J_{m}}\left(\left\{\gamma^{p}, M^{p}\right\}\right) .
$$

We note that $\sum_{m=1}^{r} T_{J_{m}}=T_{0}$.

We fix $m$, hereafter. We write the coordinate expression $\left(x_{m}^{1} \circ \gamma^{p}, \ldots, x_{m}^{n} \circ \gamma^{p}\right)$ of $\gamma^{p}$ by the same notation $\gamma^{p}$, and $\left(\left(M_{i}^{p}\right)^{1},\left(M_{i}^{p}\right)^{2}, \ldots,\left(M_{i}^{p}\right)^{n}\right)$ as $M_{i}^{p}$, unless confusion could occur. Then, $\left(\gamma^{p}, M^{p}\right)=\left(\gamma^{p},\left(M_{1}^{p}, \ldots, M_{n-1}^{p}\right)\right)$ belongs to the Hilbert space

$$
H^{2}\left(J_{m}, \boldsymbol{R}^{n}\right) \times\left(H^{1}\left(J_{m}, \boldsymbol{R}^{n}\right)\right)^{n-1} .
$$

We often denote the above Hilbert space by $H^{2} \times\left(H^{1}\right)^{n-1}$. (In the same way, we often denote the Banach space $C^{1}\left(J_{m}, \boldsymbol{R}^{n}\right) \times\left(C^{0}\left(J_{m}, \boldsymbol{R}^{n}\right)\right)^{n-1}$ by $C^{1} \times\left(C^{0}\right)^{n-1}$.) We denote by a dot the derivative with respect to $t$. Since $\left|\nabla_{t}^{\perp} M_{i}\right|^{2}=\sum_{j=1}^{n-1}\left|\left\langle\nabla_{t} M_{i}, M_{j}\right\rangle\right|^{2},\left.\mathfrak{T}\right|_{J_{m}}(\{\gamma, M\})$ has the following expression:

$$
\left.\mathfrak{T}\right|_{J_{m}}(\{\gamma, M\})=\int_{J_{m}} \Psi(\ddot{\gamma}(t), \dot{M}(t), \dot{\gamma}(t), \gamma(t), M(t)) d t,
$$

where $\gamma(t), M(t)$ are the coordinate expressions and $\Psi(\ddot{\gamma}, \dot{M}, \dot{\gamma}, \gamma, M)$ is defined as follows:

$$
\begin{aligned}
\Psi(\ddot{\gamma}, \dot{M}, \dot{\gamma}, \gamma, M)= & g_{k l}\left\{(\ddot{\gamma})^{k}+\Gamma_{r s}^{k}(\dot{\gamma})^{r}(\dot{\gamma})^{s}\right\}\left\{(\ddot{\gamma})^{l}+\Gamma_{r s}^{l}(\dot{\gamma})^{r}(\dot{\gamma})^{s}\right\} \\
& +v \sum_{i, j=1}^{n-1}\left[g_{k l}\left(M_{j}\right)^{l}\left\{\left(\dot{M}_{i}\right)^{k}+\Gamma_{r s}^{k}(\dot{\gamma})^{r}\left(M_{i}\right)^{s}\right\}\right]^{2},
\end{aligned}
$$

where $(\ddot{\gamma})^{k}$, etc. are the components of $\ddot{\gamma}$, etc., $g_{k l}=g_{k l}(\gamma)$ are the components of the Riemannian metric $g$ of $\mathcal{M}$ and the Einstein summation convention is adopted. We think of the right hand side of (2.3) as a formal functional of $(\gamma, M) \in H^{2} \times\left(H^{1}\right)^{n-1}$, and write it as

$$
\hat{\mathfrak{T}}_{J_{m}}(\gamma, M) \text {. }
$$

Since $H^{2} \times\left(H^{1}\right)^{n-1}$ is compactly embedded in $C^{1} \times\left(C^{0}\right)^{n-1}$ and $\Psi$ is quadratic with respect to $\ddot{\gamma}$ and $\dot{M}$, we obtain the following lemma.

LEMMA 2.2. The functional $\hat{\mathfrak{T}}_{J_{m}}$ is continuous with respect to the strong topology of $H^{2}\left(J_{m}, \boldsymbol{R}^{n}\right) \times\left(H^{1}\left(J_{m}, \boldsymbol{R}^{n}\right)\right)^{n-1}$.

Now, we need the following fact from [2]. Let $\mathfrak{X}$ be a Banach space. For a subset $A$ of $\mathfrak{X}$, let C.h. [A] denote the convex hull of $A$, that is, the set of all linear combinations $\sum_{s=1}^{u} \lambda_{s} X_{s}$ of elements $X_{s} \in A$, where $0 \leqslant \lambda_{s} \leqslant 1$ and $\sum_{s=1}^{u} \lambda_{s}=1$.

LemmA 2.3 (Corollary V.3.14 of [2]). Let $\mathfrak{X}$ be a Banach space. Let $\left\{X_{n}\right\}_{n=1}^{\infty}$ be a sequence of elements of $\mathfrak{X}$ converging weakly to an element $X$ of $\mathfrak{X}$. Then, some sequence of C.h. $\left[\left\{X_{n} ; n=1,2, \ldots\right\}\right]$ converges to $X$ in the metric topology. 
By applying the above lemma, we have the following

LEMMA 2.4. Let $p_{0}$ be a positive integer. Then there exists a sequence $\left\{\left(\zeta^{\alpha}, N^{\alpha}\right)\right\}_{\alpha=1}^{\infty}$ in $S\left(p_{0}\right)$ which converges to $\left(\gamma^{\infty}, M^{\infty}\right)$ as $\alpha \rightarrow \infty$ with respect to the strong topology of $H^{2}\left(J_{m}, \boldsymbol{R}^{n}\right) \times\left(H^{1}\left(J_{m}, \boldsymbol{R}^{n}\right)\right)^{n-1}$, where

$$
S\left(p_{0}\right)=\text { C.h. }\left[\left\{\left(\gamma^{p}, M^{p}\right) ; p \geqslant p_{0}\right\}\right] .
$$

We need another lemma to prove that $\left\{\gamma^{\infty}, M^{\infty}\right\}$ is a minimizer of $\mathfrak{T}$.

LEMMA 2.5. For any $\varepsilon>0$, there exists a positive integer $p_{0}$ such that

$$
\hat{\mathfrak{T}}_{J_{m}}(\zeta, N) \leqslant T_{J_{m}}+\varepsilon \text { for all }(\zeta, N) \in S\left(p_{0}\right) .
$$

PROOF. To prove this lemma, we first show the following two lemmas.

LEMMA 2.6. For any $\varepsilon>0$, there exists a positive integer $p_{0}$ such that

$$
\left|\hat{\mathfrak{T}}_{J_{m}}(\zeta, N)-\int_{J_{m}} \Psi\left(\ddot{\zeta}, \dot{N}, \dot{\gamma}^{\infty}, \gamma^{\infty}, M^{\infty}\right) d t\right| \leqslant \varepsilon \quad \text { for all } \quad(\zeta, N) \in S\left(p_{0}\right) .
$$

PROOF. Let $\varepsilon$ be an arbitrary positive number. By the convexity of an open ball in $\boldsymbol{R}^{n}$ together with the fact that $\left\{\gamma^{p}\right\}$ converges to $\gamma^{\infty}$ in the $C^{1}$ topology and $\left\{M^{p}\right\}$ converges to $M^{\infty}$ in the $C^{0}$ topology, we have the following. For any $\delta>0$, there exists a positive integer $p_{0}$ such that for all $(\zeta, N) \in S\left(p_{0}\right)$,

$$
\left|\zeta(t)-\gamma^{\infty}(t)\right|<\delta, \quad\left|\dot{\zeta}(t)-\dot{\gamma}^{\infty}(t)\right|<\delta, \quad\left|N_{i}(t)-M_{i}^{\infty}(t)\right|<\delta
$$

on $J_{m}$. Therefore, there exists a positive integer $p_{0}$ such that

$$
\begin{aligned}
& \left|\hat{\mathfrak{T}}_{J_{m}}(\zeta, N)-\int_{J_{m}} \Psi\left(\ddot{\zeta}, \dot{N}, \dot{\gamma}^{\infty}, \gamma^{\infty}, M^{\infty}\right) d t\right| \\
& \quad \leqslant \varepsilon\left(\sum_{k, l} \int_{J_{m}}\left|\ddot{\zeta}^{k} \ddot{\zeta}^{l}\right| d t+\sum_{k} \int_{J_{m}}\left|\ddot{\zeta}^{k}\right| d t+\sum_{k, l, i} \int_{J_{m}}\left|\dot{N}_{i}^{k} \dot{N}_{i}^{l}\right| d t+\sum_{k, i} \int_{J_{m}}\left|\dot{N}_{i}^{k}\right| d t\right)
\end{aligned}
$$

for all $(\zeta, N) \in S\left(p_{0}\right)$, where the components $(\ddot{\zeta})^{k},\left(\dot{N}_{i}\right)^{k}$ are simply written as $\ddot{\zeta}^{k}, \dot{N}_{i}^{k}$.

Thus, it is sufficient to show that the quantities

$$
\sum_{k, l} \int_{J_{m}}\left|\ddot{\zeta}^{k} \ddot{\zeta}^{l}\right| d t, \quad \sum_{k} \int_{J_{m}}\left|\ddot{\zeta}^{k}\right| d t, \quad \sum_{k, l, i} \int_{J_{m}}\left|\dot{N}_{i}^{k} \dot{N}_{i}^{l}\right| d t \quad \text { and } \quad \sum_{k, i} \int_{J_{m}}\left|\dot{N}_{i}^{k}\right| d t
$$

are bounded by a constant independent of $p_{0}$ and $(\zeta, N)$. Suppose that $(\zeta, N)$ is expressed as

$$
(\zeta, N)=\sum_{s=1}^{u} \lambda_{s}\left(\gamma^{q_{s}}, M^{q_{s}}\right)
$$

where $\lambda_{1}, \ldots, \lambda_{u}$ are non-negative real numbers such that $\sum_{s=1}^{u} \lambda_{s}=1$, and $q_{1}, \ldots, q_{u}$ are positive integers. Then,

$$
\sum_{k, l=1}^{n}\left|\ddot{\zeta}^{k} \ddot{\zeta}^{l}\right|=\left(\sum_{k=1}^{n}\left|\ddot{\zeta}^{k}\right|\right)^{2} \leqslant n|\ddot{\zeta}|_{E}^{2} \leqslant n \max _{1 \leqslant s \leqslant u}\left|\ddot{\gamma}^{q_{s}}\right|_{E}^{2}
$$


Since $\int_{J_{m}}\left|\ddot{\gamma}^{p}\right|_{E}^{2} d t$ is bounded with respect to $p$,

$$
\sum_{k, l=1}^{n} \int_{J_{m}}\left|\ddot{\zeta}^{k} \ddot{\zeta}^{l}\right| d t
$$

is bounded by a constant independent of $p_{0}$ and $(\zeta, N)$.

Next, we consider $\sum_{k=1}^{n} \int_{J_{m}}\left|\ddot{\zeta}^{k}\right| d t$. By the Schwarz inequality,

$$
\left(\sum_{k=1}^{n} \int_{J_{m}}\left|\ddot{\zeta}^{k}\right| d t\right)^{2} \leqslant n\left|J_{m}\right| \int_{J_{m}}|\ddot{\zeta}|_{E}^{2} d t
$$

where $\left|J_{m}\right|$ is the length of the interval $J_{m}$. By the same argument as above, the right hand side of the above expression is bounded by a constant independent of $p_{0}$ and $(\zeta, N)$. In the same way, we can check that $\sum_{k, l, i} \int_{J_{m}}\left|\dot{N}_{i}^{k} \dot{N}_{i}^{l}\right| d t$ and $\sum_{k, i} \int_{J_{m}}\left|\dot{N}_{i}^{k}\right| d t$ are both bounded by a constant independent of $p_{0}$ and $(\zeta, N)$.

LEMMA 2.7. The function $\Psi$ defined by (2.4) is a convex function with respect to $\ddot{\gamma}$ and $\dot{M}$. That is, the $n^{2}$-variable function $\Psi(\mathbf{w}, \dot{\gamma}, \gamma, M)$ of $\mathbf{w}=(\ddot{\gamma}, \dot{M}) \in \boldsymbol{R}^{n^{2}}$ satisfies

$$
\Psi\left(\sum_{s=1}^{u} \lambda_{s} \mathbf{w}_{s}, \dot{\gamma}, \gamma, M\right) \leqslant \sum_{s=1}^{u} \lambda_{s} \Psi\left(\mathbf{w}_{s}, \dot{\gamma}, \gamma, M\right)
$$

for every $\mathbf{w}_{1}, \ldots, \mathbf{w}_{u} \in \boldsymbol{R}^{n^{2}}$ and real numbers $\lambda_{1}, \ldots, \lambda_{u} \geqslant 0$ such that $\sum_{s=1}^{u} \lambda_{s}=1$.

PROOF. It is sufficient to show that the Hessian $\left(\partial^{2} \Psi / \partial w^{k} \partial w^{l}\right)_{1 \leqslant k, l \leqslant n^{2}}$, where $\mathbf{w}=$ $\left(w^{1}, \ldots, w^{n^{2}}\right)$, is positive semi-definite at all points in $\boldsymbol{R}^{n^{2}}$. Since the metric tensor $\left(g_{k l}\right)$ is positive definite, it is sufficient to show that the matrix $\left(c_{k \alpha}\right)_{1 \leqslant k, \alpha \leqslant n}$ defined by

$$
c_{k \alpha}:=\sum_{j=1}^{n-1} \sum_{l, \beta=1}^{n} g_{k l} g_{\alpha \beta}\left(M_{j}\right)^{l}\left(M_{j}\right)^{\beta}
$$

is positive semi-definite. For any $\left(y^{1}, \ldots, y^{n}\right) \in \boldsymbol{R}^{n}$,

$$
\sum_{k, \alpha=1}^{n} y^{k} c_{k \alpha} y^{\alpha}=\sum_{j=1}^{n-1} \sum_{k, l, \alpha, \beta=1}^{n}\left\{y^{k} g_{k l}\left(M_{j}\right)^{l}\right\}\left\{y^{\alpha} g_{\alpha \beta}\left(M_{j}\right)^{\beta}\right\}=\sum_{j=1}^{n-1}\left\{\sum_{k, l=1}^{n} y^{k} g_{k l}\left(M_{j}\right)^{l}\right\}^{2} \geqslant 0 .
$$

Thus, $\left(c_{k \alpha}\right)$ is positive semi-definite.

Now, we prove Lemma 2.5 . Let $\varepsilon$ be an arbitrary positive number. Let $p_{0}$ be a positive integer satisfying the expression in Lemma 2.6 and $\hat{\mathfrak{T}}_{J_{m}}\left(\gamma^{p}, M^{p}\right) \leqslant T_{J_{m}}+\varepsilon$ for any integer $p \geqslant p_{0}$. Let $(\zeta, N)$ be an element of $S\left(p_{0}\right)$ expressed as $(2.5)$, where $\lambda_{1}, \ldots, \lambda_{u}$ are nonnegative real numbers such that $\sum_{s=1}^{u} \lambda_{s}=1$, and $q_{1}, \ldots, q_{u}$ are integers greater than or 
equal to $p_{0}$. Then, by Lemma 2.6 and Lemma 2.7, we have

$$
\begin{aligned}
\hat{\mathfrak{T}}_{J_{m}}(\zeta, N) & =\int_{J_{m}} \Psi(\ddot{\zeta}, \dot{N}, \dot{\zeta}, \zeta, N) d t \\
& \leqslant \int_{J_{m}} \Psi\left(\ddot{\zeta}, \dot{N}, \dot{\gamma}^{\infty}, \gamma^{\infty}, M^{\infty}\right) d t+\varepsilon \\
& \leqslant \sum_{s=1}^{u} \lambda_{s} \int_{J_{m}} \Psi\left(\ddot{\gamma}^{q_{s}}, \dot{M}^{q_{s}}, \dot{\gamma}^{\infty}, \gamma^{\infty}, M^{\infty}\right) d t+\varepsilon \\
& \leqslant \sum_{s=1}^{u} \lambda_{s} \int_{J_{m}} \Psi\left(\ddot{\gamma}^{q_{s}}, \dot{M}^{q_{s}}, \dot{\gamma}^{q_{s}}, \gamma^{q_{s}}, M^{q_{s}}\right) d t+2 \varepsilon \\
& =\sum_{s=1}^{u} \lambda_{s} \hat{\mathfrak{T}}_{J_{m}}\left(\gamma^{q_{s}}, M^{q_{s}}\right)+2 \varepsilon \\
& \leqslant T_{J_{m}}+3 \varepsilon .
\end{aligned}
$$

Thus, we complete the proof of Lemma 2.5 .

Thus, we have the following proposition.

PROPOSITION $\left.2.8 \mathfrak{T}\right|_{J_{m}}\left(\left\{\gamma^{\infty}, M^{\infty}\right\}\right) \leqslant T_{J_{m}}(1 \leqslant m \leqslant r)$.

Proof. Lemma 2.5 and Lemma 2.4 imply the following. For any $\varepsilon>0$, there exist a positive integer $p_{0}$ and a sequence $\left\{\left(\zeta^{\alpha}, N^{\alpha}\right)\right\}_{\alpha=1}^{\infty}$ in $S\left(p_{0}\right)$ such that

$$
\hat{\mathfrak{T}}_{J_{m}}\left(\zeta^{\alpha}, N^{\alpha}\right) \leqslant T_{J_{m}}+\varepsilon
$$

and $\left(\zeta^{\alpha}, N^{\alpha}\right)$ converges to $\left(\gamma^{\infty}, M^{\infty}\right)$ as $\alpha \rightarrow \infty$ with respect to the strong topology. Thus, Lemma 2.2 yields that

$$
\hat{\mathfrak{T}}_{J_{m}}\left(\zeta^{\alpha}, N^{\alpha}\right) \rightarrow \hat{\mathfrak{T}}_{J_{m}}\left(\gamma^{\infty}, M^{\infty}\right) \quad \text { as } \quad \alpha \rightarrow \infty .
$$

Therefore, by (2.6), we have $\hat{\mathfrak{T}}_{J_{m}}\left(\gamma^{\infty}, M^{\infty}\right) \leqslant T_{J_{m}}+\varepsilon$. Hence, $\hat{\mathfrak{T}}_{J_{m}}\left(\gamma^{\infty}, M^{\infty}\right) \leqslant T_{J_{m}}$.

Proposition 2.8 implies that $\mathfrak{T}\left(\left\{\gamma^{\infty}, M^{\infty}\right\}\right)=T_{0}$, because

$$
\mathfrak{T}\left(\left\{\gamma^{\infty}, M^{\infty}\right\}\right)=\left.\sum_{m=1}^{r} \mathfrak{T}\right|_{J_{m}}\left(\left\{\gamma^{\infty}, M^{\infty}\right\}\right) \leqslant \sum_{m=1}^{r} T_{J_{m}}=T_{0} .
$$

That is, $\left\{\gamma^{\infty}, M^{\infty}\right\} \in \mathcal{U} \mathcal{C}_{0}(l, \varphi)$ attains the infimum $T_{0}$ of the energy $\mathfrak{T}$.

3. Regularity of minimizers. We shall show the regularity of a minimizer $\{\gamma, M\} \in$ $\mathcal{U C}_{0}(l, \varphi)$. Let $\mathcal{C}_{0}(l, \varphi)$ be the $C^{1,0}$ connected component of $\mathcal{C}(l, \varphi)$ including $\{\gamma, M\}$. If we reparameterize an element of $\mathcal{C}_{0}(l, \varphi)$ by arc length, then the resulting element of $\mathcal{U C}(l, \varphi)$ actually belongs to $\mathcal{U C}_{0}(l, \varphi)$. Therefore, $\{\gamma, M\}$ also minimizes the energy $\mathfrak{T}$ in $\mathcal{C}_{0}(l, \varphi)$.

We think of $\{\gamma, M\}$ as a "critical point" of $\mathfrak{T}$ on the space $\mathcal{C}(\varphi)$ under the constraint of the length of $\gamma$, and use the Lagrange multiplier principle. First, we define the following vector 
space:

$$
T_{\{\gamma, M\}} \mathcal{C}(\varphi)=\left\{\begin{array}{ll}
\Lambda \text { is an } H^{2} \text { vector field along } \gamma: S^{1} \rightarrow \mathcal{M} \\
(\Lambda, F) ; & \text { and } F:[0, l] \rightarrow \mathfrak{s o}(n-1) \text { is an } H^{1} \text { curve satisfying } \\
& F(l)=\varphi^{-1} F(0) \varphi
\end{array}\right\},
$$

where $\mathfrak{s o}(n-1)$ stands for the vector space of all $(n-1)$-by- $(n-1)$ skew-symmetric matrices.

Lemma 3.1. Let $(\Lambda, F) \in T_{\{\gamma, M\}} \mathcal{C}(\varphi)$. Let $f_{i}^{j}(t)$ be the ji component of $F(t)$. We can construct a variation $\left\{\gamma^{\lambda}, M^{\lambda}\right\}(|\lambda| \ll 1)$ of $\{\gamma, M\}\left(=\left\{\gamma^{0}, M^{0}\right\}\right)$ in $\mathcal{C}(\varphi)$ satisfying the following. (We set $\tilde{\gamma}(\lambda, t)=\gamma^{\lambda}(t), \tilde{M}(\lambda, t)=M^{\lambda}(t)$, below.) The maps $\tilde{\gamma}, \tilde{M}$ are $C^{\infty}$ with respect to $\lambda$ for any fixed $t$, and satisfy

$$
\begin{gathered}
\frac{\partial \tilde{\gamma}}{\partial \lambda}(0, t)=\Lambda(t), \\
\left(D_{\lambda}^{\perp t} \tilde{M}_{i}\right)(0, t)=\sum_{j=1}^{n-1} f_{i}^{j}(t) M_{j}(t) \quad(1 \leqslant i \leqslant n-1) .
\end{gathered}
$$

Here, $D_{\lambda}^{\perp t} \tilde{M}_{i}$ is the normal component of the vector field $\nabla_{\lambda} \tilde{M}_{i}$ along $\gamma^{\lambda}$, that is,

$$
D_{\lambda}^{\perp t} \tilde{M}_{i}=\nabla_{\lambda} \tilde{M}_{i}-\left\langle\nabla_{\lambda} \tilde{M}_{i} \tilde{T}\right\rangle \tilde{T},
$$

where $\tilde{T}=(1 /|\partial \tilde{\gamma} / \partial t|) \partial \tilde{\gamma} / \partial t$ is the unit tangent vector to $\gamma^{\lambda}$ and $\nabla_{\lambda}=\nabla_{\partial / \partial \lambda}$.

Proof. We set $\tilde{\gamma}(\lambda, t)=\exp \lambda \Lambda(t)$, where exp is the exponential map in $\mathcal{M}$. If $|\lambda|$ is sufficiently small, $\gamma^{\lambda}$ is an $H^{2}$ regular closed curve. Let $\tilde{M}=\left(\tilde{M}_{1}, \ldots, \tilde{M}_{n-1}\right)$ be the solution of the following initial value problem of the ordinary differential equation with independent variable $\lambda$ :

$$
\nabla_{\lambda} \tilde{M}_{i}=-\left\langle\tilde{M}_{i}, \nabla_{\lambda} \tilde{T}\right\rangle \tilde{T}+\sum_{j=1}^{n-1} f_{i}^{j} \tilde{M}_{j} \quad(1 \leqslant i \leqslant n-1),
$$

$$
\tilde{M}(0, t)=M(t) .
$$

By (3.3), it is verified that $M^{\lambda}(t)$ is an orthonormal frame field of the normal bundle along $\left.\gamma^{\lambda}\right|_{[0, l]}$ and (3.2) holds. Also, we can check that $M^{\lambda}(l)=M^{\lambda}(0) \varphi$ in the following way. We define $\sigma(\lambda) \in O(n-1)$ by the relation $M^{\lambda}(l)=M^{\lambda}(0) \sigma(\lambda)$. Then, (3.3) yields that $\sigma(\lambda)$ satisfies

$$
\begin{gathered}
\frac{d \sigma(\lambda)}{d \lambda}=\sigma(\lambda) F(l)-F(0) \sigma(\lambda), \\
\sigma(0)=\varphi .
\end{gathered}
$$

Also, $F(l)=\varphi^{-1} F(0) \varphi$ yields that $\sigma(\lambda) \equiv \varphi$ satisfies (3.5) and (3.6). Therefore, $\sigma(\lambda) \equiv \varphi$.

Now, the first variation formula for $\mathfrak{T}$ is calculated as follows (cf. [5], [10]). We use the sign convention of the curvature tensor $R$ corresponding to that of [8]. 
LEMMA 3.2. Let $(\Lambda, F) \in T_{\{\gamma, M\}} \mathcal{C}(\varphi)$, and let $\left\{\gamma^{\lambda}, M^{\lambda}\right\}(|\lambda| \ll 1)$ be the variation of $\{\gamma, M\}$ in $\mathcal{C}(\varphi)$ constructed in the proof of Lemma 3.1. Then,

$$
\begin{aligned}
&\left.\frac{d}{d \lambda}\right|_{\lambda=0} \mathfrak{T}\left(\left\{\gamma^{\lambda}, M^{\lambda}\right\}\right) \\
&= \int_{0}^{l}\left[\left\langle\left(\nabla_{t}\right)^{2} \Lambda, 2 \nabla_{t} \dot{\gamma}\right\rangle\right. \\
&+\left\langle\nabla_{t} \Lambda,-\left(3\left|\nabla_{t} \dot{\gamma}\right|^{2}+v \sum_{i=1}^{n-1}\left|\nabla_{t}^{\perp} M_{i}\right|^{2}\right) \dot{\gamma}\right. \\
&\left.+2 v \sum_{i=1}^{n-1}\left(\left\langle\nabla_{t} \dot{\gamma}, M_{i}\right\rangle \nabla_{t}^{\perp} M_{i}-\left\langle\nabla_{t} \dot{\gamma}, \nabla_{t}^{\perp} M_{i}\right\rangle M_{i}\right)\right\rangle \\
&\left.+\left\langle\Lambda, 2 R\left(\nabla_{t} \dot{\gamma}, \dot{\gamma}\right) \dot{\gamma}+2 v \sum_{i=1}^{n-1} R\left(\nabla_{t}^{\perp} M_{i}, M_{i}\right) \dot{\gamma}\right\rangle\right] d t \\
&+2 v \sum_{i, j=1}^{n-1} \int_{0}^{l} \dot{f}_{i}^{j}\left\langle\nabla_{t}^{\perp} M_{i}, M_{j}\right\rangle d t .
\end{aligned}
$$

We denote by $d \mathfrak{T}_{\{\gamma, M\}}(\Lambda, F)$ the right hand side of (3.7). Then, $d \mathfrak{T}_{\{\gamma, M\}}: T_{\{\gamma, M\}} \mathcal{C}(\varphi) \rightarrow$ $\boldsymbol{R}$ is a linear functional. Now, we use the Lagrange multiplier principle. We consider the length functional $\mathfrak{L}(\{\gamma, M\})=\int_{0}^{l} v(t) d t$. The first variation formula for $\mathfrak{L}$ is given as follows:

$$
\left.\frac{d}{d \lambda}\right|_{\lambda=0} \mathfrak{L}\left(\left\{\gamma^{\lambda}, M^{\lambda}\right\}\right)=\int_{0}^{l}\left\langle\nabla_{t} \Lambda, \dot{\gamma}\right\rangle d t .
$$

We write the right hand side as $d \mathfrak{L}_{\{\gamma, M\}}(\Lambda, F)$. Then, $d \mathfrak{L}_{\{\gamma, M\}}: T_{\{\gamma, M\}} \mathcal{C}(\varphi) \rightarrow \boldsymbol{R}$ is a linear functional. We define the following vector space:

$$
T_{\{\gamma, M\}} \mathcal{C}(l, \varphi)=\left\{(\Lambda, F) \in T_{\{\gamma, M\}} \mathcal{C}(\varphi) ; d \mathfrak{L}_{\{\gamma, M\}}(\Lambda, F)=0\right\} .
$$

By the similar argument in the proof of Lemma 3.1 of [5], we have the following.

LEMMA 3.3. Suppose that $\gamma$ is not a closed geodesic in $\mathcal{M}$. Let $(\Lambda, F) \in T_{\{\gamma, M\}} \mathcal{C}(l, \varphi)$. We can construct a variation $\left\{\gamma^{\lambda}, M^{\lambda}\right\}(|\lambda| \ll 1)$ of $\{\gamma, M\}\left(=\left\{\gamma^{0}, M^{0}\right\}\right)$ in $\mathcal{C}(l, \varphi)$ such that $\tilde{\gamma}(\lambda, t)=\gamma^{\lambda}(t)$ and $\tilde{M}(\lambda, t)=M^{\lambda}(t)$ are $C^{\infty}$ in $\lambda$ for any fixed $t$ and (3.1) and (3.2) hold.

It is verified that the first variation formula (3.7) is valid for the variation $\left\{\gamma^{\lambda}, M^{\lambda}\right\}(|\lambda| \ll$ 1) constructed in Lemma 3.3. Suppose that $\gamma$ is not a closed geodesic in $\mathcal{M}$, hereafter. Then, the following holds.

LEMMA 3.4. There exists a real number $\mu_{0}$ such that

$$
\left(d \mathfrak{T}_{\{\gamma, M\}}+\mu_{0} d \mathfrak{L}_{\{\gamma, M\}}\right)(\Lambda, F)=0
$$

for all $(\Lambda, F) \in T_{\{\gamma, M\}} \mathcal{C}(\varphi)$.

Proof. For $(\Lambda, F) \in T_{\{\gamma, M\}} \mathcal{C}(l, \varphi)$, let $\left\{\gamma^{\lambda}, M^{\lambda}\right\}(|\lambda| \ll 1)$ be the variation constructed in Lemma 3.3. Note that $\left\{\gamma^{\lambda}, M^{\lambda}\right\}(|\lambda| \ll 1)$ is a variation in $\mathcal{C}_{0}(l, \varphi)$. Since $\{\gamma, M\}$ 
is a minimizer of $\mathfrak{T}$ in $\mathcal{C}_{0}(l, \varphi), d /\left.d \lambda\right|_{\lambda=0} \mathfrak{T}\left(\left\{\gamma^{\lambda}, M^{\lambda}\right\}\right)=0$. Thus, $d \mathfrak{T}_{\{\gamma, M\}}(\Lambda, F)=0$. Therefore, both $d \mathfrak{T}_{\{\gamma, M\}}$ and $d \mathfrak{L}_{\{\gamma, M\}}$ vanish on $T_{\{\gamma, M\}} \mathcal{C}(l, \varphi)$. Since $\gamma$ is not a closed geodesic in $\mathcal{M}$, there exists an $H^{2}$ vector field $\Omega$ along $\gamma$ such that $T_{\{\gamma, M\}} \mathcal{C}(\varphi)$ is expressed as the linear direct sum of $T_{\{\gamma, M\}} \mathcal{C}(l, \varphi)$ and the one-dimensional subspace spanned by $(\Omega, 0)$. Thus, (3.8) holds by setting

$$
\mu_{0}=-\frac{d \mathfrak{T}_{\{\gamma, M\}}(\Omega, 0)}{d \mathfrak{L}_{\{\gamma, M\}}(\Omega, 0)} .
$$

In consequence, we have the following lemma, which implies that the twist is uniformly distributed over the curve $\gamma$.

Lemma 3.5. Let $a_{i}^{j}(t)=\left\langle\nabla_{t}^{\perp} M_{i}, M_{j}\right\rangle$. Then, $a_{i}^{j}(t)$ is constant on $[0, l]$. Denote by $a=\left(a_{i}^{j}\right)$ the $(n-1)-b y-(n-1)$ matrix with ji components $a_{i}^{j}$. Then $a \in \mathfrak{s o}(n-1)$ and $\varphi a \varphi^{-1}=a$.

Proof. Let $(\Lambda, F) \in T_{\{\gamma, M\}} \mathcal{C}(\varphi)$ satisfy $\Lambda=0$ and the following:

$$
\begin{aligned}
& f_{i}^{j}(t) \equiv 0 \quad \text { if }(j, i) \neq(p, q) \text { and }(j, i) \neq(q, p), \\
& f_{q}^{p}(0)=f_{q}^{p}(l)=0 .
\end{aligned}
$$

Then, since $(3.8)=0$,

$$
\int_{0}^{l} \dot{f}_{q}^{p} a_{q}^{p} d t=0
$$

This holds for all $f_{q}^{p} \in H^{1}([0, l], \boldsymbol{R})$ satisfying $f_{q}^{p}(0)=f_{q}^{p}(l)=0$. Therefore, by the du Bois-Reymond lemma ([13]), $a_{q}^{p}(t)$ is constant on $[0, l]$.

By the Leibniz rule, $a \in \mathfrak{s o}(n-1)$. We show $\varphi a \varphi^{-1}=a$. Let $(\Lambda, F) \in T_{\{\gamma, M\}} \mathcal{C}(\varphi)$ satisfy $\Lambda=0$. Then, by the relation $F(l)=\varphi^{-1} F(0) \varphi$, the left hand side of (3.8) equals to

$$
2 v \operatorname{tr}(a F(l)-a F(0))=2 v \operatorname{tr}\left(F(0)\left(\varphi a \varphi^{-1}-a\right)\right) .
$$

This vanishes for all $F(0) \in \mathfrak{s o}(n-1)$. Therefore, $\varphi a \varphi^{-1}=a$.

Since $\varphi a \varphi^{-1}=a$, (3.8) reduces to the following:

$$
\begin{aligned}
& \int_{0}^{l}\left[\left\langle\left(\nabla_{t}\right)^{2} \Lambda, 2 \nabla_{t} \dot{\gamma}\right\rangle\right. \\
& +\left\langle\nabla_{t} \Lambda,-\left(3\left|\nabla_{t} \dot{\gamma}\right|^{2}-\mu_{0}+v \sum_{i=1}^{n-1}\left|\nabla_{t}^{\perp} M_{i}\right|^{2}\right) \dot{\gamma}\right. \\
& \left.\quad+2 v \sum_{i=1}^{n-1}\left(\left\langle\nabla_{t} \dot{\gamma}, M_{i}\right\rangle \nabla_{t}^{\perp} M_{i}-\left\langle\nabla_{t} \dot{\gamma}, \nabla_{t}^{\perp} M_{i}\right\rangle M_{i}\right)\right\rangle \\
& \left.+\left\langle\Lambda, 2 R\left(\nabla_{t} \dot{\gamma}, \dot{\gamma}\right) \dot{\gamma}+2 v \sum_{i=1}^{n-1} R\left(\nabla_{t}^{\perp} M_{i}, M_{i}\right) \dot{\gamma}\right\rangle\right] d t=0 .
\end{aligned}
$$


We show that $\{\gamma, M\}$ is $C^{\infty}$ on $(0, l)$. Let $I=\left(t_{1}, t_{2}\right) \subset(0, l)$ be an open interval, and $\left(U,\left(x^{j}\right)\right)$ a local coordinate neighborhood of $\mathcal{M}$ such that $\gamma\left(\left[t_{1}, t_{2}\right]\right) \subset U$. We show that the coordinate expressions of $\gamma$ and $M$ are of class $C^{\infty}$ on $I$. Let $\Lambda^{k}$ be the component of $\Lambda$ with respect to the basis $\left(\partial / \partial x^{1}, \ldots, \partial / \partial x^{n}\right)$. We use the Einstein convention in the following. The integrand of (3.10) is expressed as follows:

$$
\ddot{\Lambda}^{k} P_{k}+\dot{\Lambda}^{k} Q_{k}+\Lambda^{k} S_{k},
$$

where

$$
P_{k}=2 g_{k l}\left(\ddot{\gamma}^{l}+\Gamma_{p q}^{l} \dot{\gamma}^{p} \dot{\gamma}^{q}\right) .
$$

We omit the explicit expressions of $Q_{k}$ and $S_{k}$, although they are expressed by $\ddot{\gamma}^{p}, \dot{\gamma}^{p}, \gamma^{p}$, $\dot{M}^{p}, M^{p}, g_{k l}, \Gamma_{p q}^{l}$ and the curvature tensor $R_{j k l m}$.

Now, we state the following du Bois-Reymond type lemma, whose proof is omitted.

LEMMA 3.6. Let $I=\left(t_{1}, t_{2}\right)$ be a finite open interval. Let $f, g, h \in L^{1}(I)$. If

$$
\int_{t_{1}}^{t_{2}}\left(\psi^{\prime \prime} f+\psi^{\prime} g+\psi h\right) d t=0
$$

for all $\psi \in C_{0}^{\infty}(I)$, then $f \in W^{1,1}(I), f^{\prime}-g \in W^{1,1}(I)$ and

$$
\left(f^{\prime}-g\right)^{\prime}+h=0 \text {. }
$$

We simply write $W^{1,1}(I)$, etc. as $W^{1,1}$, etc. By Lemma 3.6, $P_{k}, P_{k}^{\prime}-Q_{k} \in W^{1,1}$ and

$$
\left(P_{k}^{\prime}-Q_{k}\right)^{\prime}+S_{k}=0 .
$$

We show, by induction, that

$$
\gamma^{l} \in W^{r+1,1}, \quad M^{l} \in W^{r, 1}
$$

for any integer $r \geqslant 1$. First, (3.12) is obvious if $r=1$. Assume that (3.12) holds. Since the matrix $\left(g_{l k}\right)$ is invertible, $\ddot{\gamma}^{l}$ is expressed as

$$
\ddot{\gamma}^{l}=\frac{1}{2} g^{l k} P_{k}-\Gamma_{p q}^{l} \dot{\gamma}^{p} \dot{\gamma}^{q},
$$

where $\left(g^{l k}\right)$ is the inverse matrix of $\left(g_{l k}\right)$. By (3.12), $P_{k}, Q_{k}$ and $S_{k} \in W^{r-1,1}$. Thus, (3.11) yields $P_{k} \in W^{r, 1}$. Therefore, the right hand side of (3.13) belongs to $W^{r, 1}$, and so $\gamma^{l} \in$ $W^{r+2,1}$ follows. Now, by the Leibniz rule,

$$
\left(\nabla_{t}^{\perp} M_{i}\right)^{k}=\dot{M}_{i}^{k}+\dot{\gamma}^{l} \Gamma_{l m}^{k} M_{i}^{m}+\left\langle\nabla_{t} \dot{\gamma}, M_{i}\right\rangle \dot{\gamma}^{k} .
$$

Since $\gamma^{l} \in W^{r+2,1}$, the second and third terms of the right hand side of (3.14) are $W^{r, 1}$. Also, by Lemma 3.5, the left hand side of (3.14) is $W^{r, 1}$. Therefore, $M_{i}^{k} \in W^{r+1,1}$. Thus, (3.12) holds for any integer $r \geqslant 1$, and hence $\gamma^{l}, M^{l} \in C^{\infty}$.

Next, we show that $\gamma$ is $C^{\infty}$ on the whole $S^{1}$, and $M$ is also $C^{\infty}$ on $[0, l]$. Let $I=\left(t_{1}, t_{2}\right)$ be an open interval, where $-l<t_{1}<0<t_{2}<l$, and let $\left(U,\left(x^{j}\right)\right)$ be a local coordinate 
neighborhood of $\mathcal{M}$ such that $\gamma\left(\left[t_{1}, t_{2}\right]\right) \subset U$. Let $\hat{M}$ be the orthonormal frame of the normal bundle along $\left.\gamma\right|_{\left[t_{1}, t_{2}\right]}$ given by

$$
\begin{aligned}
& \hat{M}(t)=M(t+l) \varphi^{-1} \quad \text { if } t_{1} \leqslant t<0, \\
& \hat{M}(t)=M(t) \quad \text { if } 0 \leqslant t \leqslant t_{2} .
\end{aligned}
$$

Note that $\hat{M}$ is of class $H^{1}$. Denote by $K(t)$ the integrand of (3.10), and by $\hat{K}(t)$ the expression which is obtained by replacing all $M_{i}$ in $K(t)$ with $\hat{M}_{i}$. Then, we can check that $K(t)=\hat{K}(t)$ for almost every $t \in I$. Thus,

$$
\int_{I} \hat{K}(t) d t=0
$$

for all $\Lambda$ whose support is contained in $I$. Let $\hat{a}_{i}^{j}(t)=\left\langle\nabla_{t}^{\perp} \hat{M}_{i}, \hat{M}_{j}\right\rangle$, and let $\hat{a}(t)$ be the matrix with $j i$ component $\hat{a}_{i}^{j}(t)$. By $a=\varphi a \varphi^{-1}$, it follows that $\hat{a}(t)=a$ for almost every $t \in I$. That is, the fact analogous to Lemma 3.5 holds. Thus, by (3.15) together with the similar argument as above, it follows that the coordinate expressions of $\left.\gamma\right|_{I}$ and $\left.\hat{M}\right|_{I}$ are $C^{\infty}$. Therefore, $\gamma$ is $C^{\infty}$ on the whole $S^{1}$, and $M$ is also $C^{\infty}$ on $[0, l]$.

Finally, we consider the case that $\gamma$ is a closed geodesic. As is well-known, $\gamma$ is $C^{\infty}$, and hence it is sufficient to show that $M$ is $C^{\infty}$ on $[0, l]$. Note that Lemma 3.1 and Lemma 3.2 still follow. Now, let $(\Lambda, F) \in T_{\{\gamma, M\}} \mathcal{C}(\varphi)$ satisfy $\Lambda=0$. Then, the variation $\left\{\gamma^{\lambda}, M^{\lambda}\right\}(|\lambda| \ll 1)$ constructed in Lemma 3.1 is, indeed, a variation in $\mathcal{C}_{0}(l, \varphi)$, because $\gamma^{\lambda}=\gamma$ for all $\lambda$. Since $\{\gamma, M\}$ is a minimizer of $\mathfrak{T}$ in $\mathcal{C}_{0}(l, \varphi)$, Lemma 3.2 yields that the second term of the right hand side of (3.7) equals to 0 for all $H^{1}$ curve $F:[0, l] \rightarrow \mathfrak{s o}(n-1)$ such that $F(l)=\varphi^{-1} F(0) \varphi$. Thus, Lemma 3.5 still holds. Therefore, by using the expression (3.14), we can see that $M$ is $C^{\infty}$ on $(0, l)$ in the same way. Also, it is shown that $\hat{M}$ is $C^{\infty}$ on $[0, l]$ in the same way as above. This completes the proof of the regularity.

\section{REFERENCES}

[ 1 ] S. S. Antman, Ordinary differential equations of nonlinear elasticity, II, Existence and regularity theory for conservative boundary value problem, Arch. Rational Mech. Anal. 61 (1976), 353-393.

[2] N. Dunford AND J. T. SChwARTZ, Linear operators, Part I, Interscience, New York, 1958.

[3] I. M. GeL'FAnd AND S. V. Fomin, Calculus of variations, Prentice-Hall, 1963.

[4] T. IVEY AND D. SInger, Knot types, homotopies and stability of closed elastic rods, Proc. London Math. Soc. (3) 79 (1999), 429-450.

[ 5] S. KAWAKUBO, Stability and bifurcation of circular Kirchhoff elastic rods, Osaka J. Math. 37 (2000), 93-137.

[ 6] S. Kawakubo, Errata to "Stability and bifurcation of circular Kirchhoff elastic rods by Satoshi Kawakubo, Osaka J. Math. 37 (2000), 93-137", Osaka J. Math. 37 (2000), 525.

[ 7 ] J. L. Kelly, General Topology, Van Nostrand, 1955.

[ 8] S. Kobayashi AND K. NomizU, Foundations of differential geometry, I, II, Interscience, $1963,1969$.

[ 9 ] N. KoIso, Elasticae in a Riemannian submanifold, Osaka J. Math. 29 (1992), 539-543.

[10] J. LANGER AND D. SINGER, The total squared curvature of closed curves, J. Differential Geom. 20 (1984), $1-22$.

[11] J. LANGer And D. Singer, Curve-straightening in Riemannian manifolds, Ann. Global Anal. Geom. 5 (1987), 133-150.

[12] J. LANGER AND D. SINGER, Lagrangian aspects of the Kirchhoff elastic rod, SIAM Rev. 38 (1996), 605-618.

[13] C. B. MorRey, Jr., Multiple integrals in the calculus of variations, Springer-Verlag, New York, 1966. 
DEPARTMENT OF MATHEMATICS

GRADUATE SCHOOL OF SCIENCE

OSAKA UNIVERSITY

1-1 MACHIKANEYAMA-CHO, TOYONAKA

OSAKA 560-0043

JAPAN

E-mail address: satoshi@math.sci.osaka-u.ac.jp 